\title{
锆-苯炔参与的金属杂环的形成及其在有机合成中的应用
}

\author{
毛可涁 ${ }^{a}$ 付晓平 $b$ 刘 丹*, ${ }^{*}$ 李 石 ${ }^{b}$ 刘元红*,b \\ ( ${ }^{a}$ 沈阳化工大学化学工程学院 沈阳 110142) \\ $\left({ }^{b}\right.$ 中国科学院上海有机化学研究所 金属有机化学国家重点实验室 上海 200032)
}

\begin{abstract}
摘要 铅-苯炔是一类非常重要且具有高度反应活性的金属有机配合物. 其与烯、炔、醛、酮、腈等不饱和底物反应时, 通过调节底物的取代基、化学计量比、配体结构以及反应条件等，可以得到多种形式的铅杂环类化合物. 该铅杂环进 一步与亲电试剂反应可以构筑结构丰富多样的有机化合物. 对铅-苯炔的合成方法及其参与的金属杂环的形成与后续 反应等方面进行了综述, 并对锆-苯炔在有机合成化学领域的发展前景进行了展望.
\end{abstract}

关键词 锆-苯炔; 金属杂环; 反应性能; 偶联反应

\section{Construction of Metallacycles via Zirconium-Benzyne Complexes and Its Application in Organic Synthesis}

\author{
Mao, Kebin ${ }^{a} \quad \mathrm{Fu}, \mathrm{Xiaoping}^{b} \quad$ Liu, Dan ${ }^{*, a} \quad$ Li, Shi ${ }^{b} \quad$ Liu, Yuanhong ${ }^{*, b}$ \\ $\left({ }^{a}\right.$ Colledge of Chemical Engineering, Shenyang University of Chemical Technology, Shenyang 110142) \\ ( ${ }^{b}$ State Key Laboratory of Organometallic Chemistry, Shanghai Institute of Organic Chemistry, \\ Chinese Academy of Sciences, Shanghai 200032)
}

\begin{abstract}
Zirconium-benzyne complex is an important and highly reactive organometallic species. It can react with unsaturated substrates such as alkenes, alkynes, aldehydes, ketones, nitriles etc. to form metallacyles. A large variety of metallacycles can be constructed by modifying the substitution patterns of the substrates, chemical ratio of reactants, different $\mathrm{Cp}$ ligands or reaction conditions and so on. These metallacycles are highly useful for further transformations through the reactions with different electrophiles. This review summarizes the synthetic methods of zirconium-benzyne complexes, reactions of zirconium-benzyne complexes with various unsaturated molecules leading to metallacycles, and the applications of these metallacycles in organic chemistry. An outlook of zirconium-benzyne complexes in organic synthesis is also provided at the end of this review.
\end{abstract}

Keywords zirconium-benzyne; metallacycle; reactivity; coupling reaction

苯炔又称脱氢苯, 可以看作苯环的邻位脱除一分子 氢而形成, 是一类非常重要的具有高反应活性的有机合 成中间体 ${ }^{[1]}$. 苯炔分子中碳碳参键的两个碳原子趋向于 $\mathrm{sp}^{2}$ 杂化, 累积烯和双自由基是其主要的共振形式(图 1). 从苯炔的电子结构图中可以看出, 由这两个 $\mathrm{sp}^{2}$ 杂化轨 道从侧面微弱地重叠所形成的 $\pi$ 键位于环平面上, 并与 苯环原有的 $\pi$ 体系相互垂直. 由于两个 $\mathrm{sp}^{2}$ 轨道相距较 远不能较好地重叠, 使得这个 $\pi$ 键十分脆弱且具有较大 的张力, 因此苯炔的性质非常活泼, 很不稳定. 苯炔可 以参与多种类型的反应，如极性或协同的环加成反应、
与亲核试剂的反应、过渡金属催化的反应以及苯炔自身 的聚合等, 因此其作为有机活性中间体在物理有机化 学、有机合成化学及天然产物合成等领域备受关注.

苯炔本身活性太高不易分离获得，所以通常都是用 其合成前体代替，在反应过程中原位产生苯炔中间体. 有机合成中常见的苯炔前体主要有以下几种(Scheme $1)^{[2 a]}$. 由邻氨基苯甲酸制成的重氮盐 $\mathbf{1}^{[2 b]}$ 加热分解即得 苯炔，但是该前体对碰撞敏感，爆炸性强，难于分离. 苯炔前体 $\mathbf{2}^{[2 \mathrm{c}]}$ 和 $\mathbf{3}^{[2 \mathrm{~d}]}$ 制备较简单，但是必须在强碱作用 下才能发生消除反应得到苯炔. 前体 $4^{[2 \mathrm{e}]}$ 可通过邻卤代

\footnotetext{
*E-mail: yhliu@mail.sioc.ac.cn

Received November 13, 2012; revised November 26, 2012; published online December 7, 2012.

Project supported by the National Natural Science Foundation of China (Nos. 21072208, 21125210, 21121062) and the Major State Basic Research Development Program (973 Program, No. 2011CB808700).

国家自然科学基金(Nos. 21072208, 21125210, 21121062)和国家重点基础研究发展计划(973 计划, No. 2011CB808700)资助项目.
} 

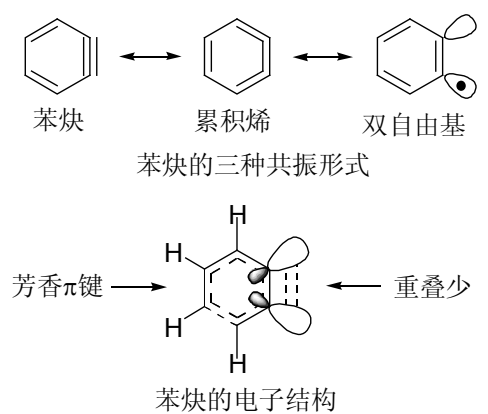

图 1 苯炔的结构

Figure 1 The structure of benzyne

氟苯在强碱作用下得到, 但其极不稳定. 前体 $\mathbf{5}^{[2 \mathrm{ff}]}$ 需在 氧化剂如四醋酸铅的存下才可以转化为苯炔. 前体 $6^{[2 \mathrm{~g}]}$ 和 $7^{[2 \mathrm{~h}]}$ 皆可在温和的条件下 (在 $\mathrm{F}^{-}$离子存在下) 定量地转 化为苯炔, 但化合物 $\mathbf{6}$ 的制备操作繁琐，原料昂贵，而 化合物 7 的合成相对简单, 是目前应用最为广泛的一类 苯炔前体. 二芳基二茂锆 8 是含有前过渡金属锆的一种 苯炔前体, 在加热的条件下可以经过 $\beta$ - $\mathrm{H}$ 消除反应现场 生成苯炔 ${ }^{[3]}$, 同时释放出一分子芳烃, 所形成的苯炔分 子通过与低价锆的配位作用生成较为稳定的金属-苯炔 配合物, 如锆-苯炔配合物 9 的形成(Scheme 2). 其中 锆-苯炔的结构可用金属-炔烃配合物与锆杂三元环的 共振形式来表示.<smiles>Nc1ccccc1C(=O)O</smiles>

1<smiles>Nn1nnc2ccccc21</smiles>
5<smiles>Brc1ccccc1Br</smiles>

2<smiles>CS(=O)(=O)c1ccccc1I(O)c1ccccc1</smiles><smiles>FC(F)(F)Oc1cccc(Br)c1</smiles>

3<smiles>CCOc1ccccc1C</smiles>

7

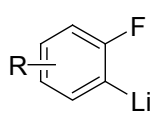

4

Scheme 1

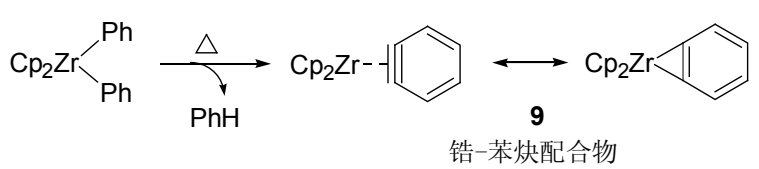

Scheme 2

锆-苯炔配合物与烯烃、炔烃、醛、酮、腈等均可 以发生偶联反应形成在有机合成中非常重要的五元金 属杂环类化合物. 该金属杂环在特定的反应条件下可以 与多种多样的亲电试剂进一步发生反应, 从而使金属杂 环中的锆-碳键得到选择性的修饰与活化, 淬灭后生成 进一步官能团化的产物. 本文就锆-苯炔的合成方法及 其参与的金属杂环的形成与后续反应等方面进行总结 与探讨.

\section{1 锆-苯炔的合成方法}

\section{1 以二芳基二茂锆为前体合成锆-苯炔配合物}

锆-苯炔可以由二芳基二茂锆在加热的条件下经 $\beta-\mathrm{H}$ 消除反应现场生成，因此二芳基二茂锆的合成就显 得尤为重要. 其中二苯基二茂锆最早由 Rausch 小组 ${ }^{[4]}$ 于 1973 年通过二氯二茂锆与苯基锂在低温下反应制得, 其结构于 1998 年通过 X 射线单晶衍射分析得到了进一 步的确定 ${ }^{[5]}$. 该配合物在室温下惰性气体环境中可长期 保存, 在空气中短时间内能稳定存在, 但在真空下加热 到 $130{ }^{\circ} \mathrm{C}$ 以上时会产生分解. 与此同时, 他们还制备了 其它几种类似的二芳基茂类金属配合物如: $\mathrm{Cp}_{2} \mathrm{Hf}\left(\mathrm{C}_{6}\right.$ $\left.\mathrm{H}_{5}\right)_{2}, \operatorname{Ind}_{2} \mathrm{Zr}\left(\mathrm{C}_{6} \mathrm{H}_{5}\right)_{2}, \operatorname{Ind}_{2} \operatorname{Ti}\left(\mathrm{C}_{6} \mathrm{H}_{5}\right)_{2}, \operatorname{Ind}_{2} \operatorname{Ti}\left(\mathrm{C}_{6} \mathrm{~F}_{5}\right)_{2}$ (Ind $=$ Indenyl)和 5,5-bis( $\pi$-indenyl)dibenzotitanole. 其中, 狮基 金属配合物较简单 $\mathrm{Cp}$ 作为配体的配合物更加稳定. 不 过, 首先发现二苯基二茂锆可以转化为锆-苯炔的则是 德国的 Erker 小组. 1977 年, Erker 小组 ${ }^{[6]}$ 发现在 $20{ }^{\circ} \mathrm{C}$ 光 照二芳基二茂锆 10 或 12 的苯溶液可以使其有效地发 生还原消除反应，以几乎定量的收率分别生成联苯类化 合物 11 与 13 (Scheme 3). 利用该方法他们仔细分析了 一系列二芳基二茂锆在芳香性溶剂苯或甲苯中的热解 反应产物. 即热解反应结束后接着进行光解反应, 通过 光解产物分析推测体系中可能生成的二芳基二茂锆物 种. 通过该实验他们发现芳香性溶剂可以参与反应. 例 如，当双(对甲基苯基)二茂锆 12 在苯中 $70{ }^{\circ} \mathrm{C}$ 加热至 $9 \mathrm{~h}$ 时, 可以得到芳香环偶联产物 13 $\sim 16$ (Eq. 1). 对于 14 16 的生成, Erker 认为反应中首先生成了锆-苯炔中间体 17, 其与溶剂苯发生 $\mathrm{C}-\mathrm{H}$ 键插入反应生成 18 和 19. 此 外, 锆-苯炔 9 可由 18 和 19 转化得到, 9 继续与苯反应 得到二苯基二茂锆. 18, 19 和二苯基二茂锆经光照后得 到相应的偶联产物 14 16 (Scheme 4). 在此文中 Erker 认为苯炔的生成可能并非经历 $\beta-\mathrm{H}$ 消除反应，而是由二 芳基二茂锆中其中一个芳基擅取另一个芳基的邻位质 子所致.

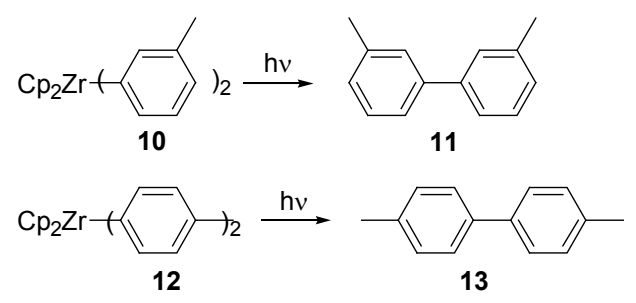

Scheme 3

1986 年, Buchwald 小组 ${ }^{[7]}$ 通过在反应体系中加入三 甲基膦配体, 成功地捕获到三甲基膦所稳定的二茂锆苯炔配合物 20, 其结构通过 X 射线单晶衍射分析得到 确认(Scheme 5). 


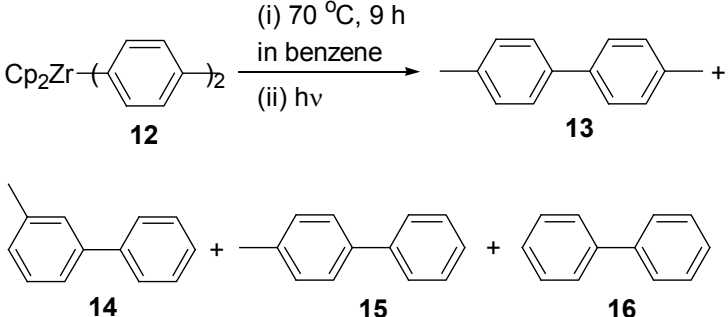

15

16

ratio of $13: 14: 15: 16=1.49: 1: 1.12: 1.09$

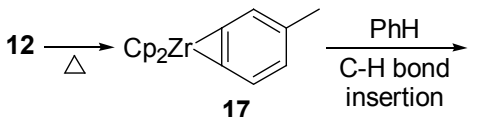<smiles>Cc1ccc([Te](=[W])c2ccccc2)cc1</smiles>
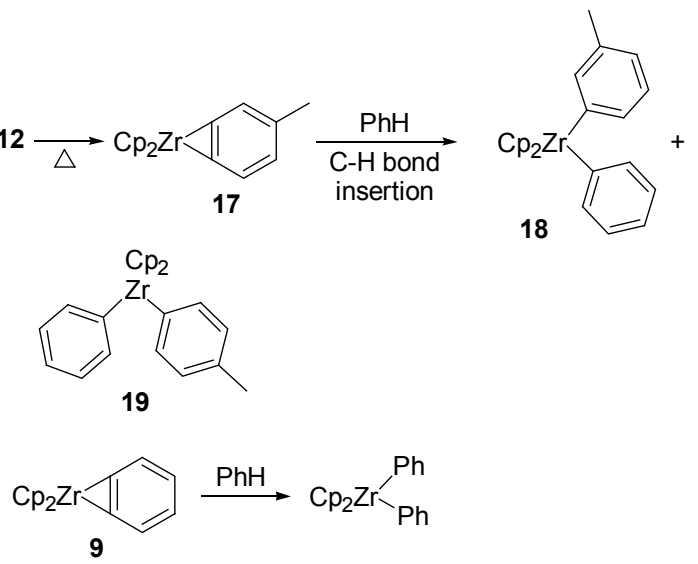

Scheme 4

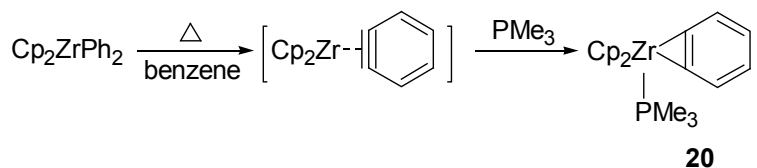

Scheme 5

\section{2 以 $\mathrm{Cp}_{2} \mathrm{Zr}(\mathrm{R}) \mathrm{Cl}$ 为前体合成锆 - 苯炔配合物}

Buchwald 小组首先发展了以 $\mathrm{Cp}_{2} \mathrm{Zr}(\mathrm{Me}) \mathrm{Cl}$ 为前体制 备锆-苯炔的方法. $\mathrm{Cp}_{2} \mathrm{Zr}(\mathrm{Me}) \mathrm{Cl}$ 与芳基锂 21 反应得到甲 基/芳基二茂锆 22, 其在加热条件下释放出一分子甲烷 的同时, 能够原位生成锆-苯炔 23 (Scheme 6) ${ }^{[8 \sim 12]}$. Buchwald 小组发展的另一种锆-苯炔前体是 $\mathrm{Cp}_{2} \mathrm{Zr}$ $(i-\mathrm{Bu}) \mathrm{Cl}$. 二氯二茂锆在叔丁基锂 ${ }^{[13]}$ 或叔丁基氯化镁 ${ }^{[14]}$ 的作用下经取代、 $\beta$ - $\mathrm{H}$ 消除和插入反应现场生成 $\mathrm{Cp}_{2} \mathrm{Zr}(i-\mathrm{Bu}) \mathrm{Cl}$ ，其与芳基锂发生盐消除反应得到异丁 基/芳基锆 24, 经 $\beta-\mathrm{H}$ 消除脱除异丁烷的同时生成锆-苯 炔 23 (Scheme 6) ${ }^{[13]}$. 上述两种方法的缺点是前体 $\mathrm{Cp}_{2} \mathrm{Zr}$ $(\mathrm{Me}) \mathrm{Cl}$ 或者 $\mathrm{Cp}_{2} \mathrm{Zr}(i-\mathrm{Bu}) \mathrm{Cl}$ 的制备较为困难或者繁琐. 而 $\mathrm{Cp}_{2} \mathrm{ZrPh}_{2}$ 可用简便易得的原料 $\mathrm{Cp}_{2} \mathrm{ZrCl}_{2}$ 和苯基锂直 接反应得到.

此外, Buchwald 等 ${ }^{[8]}$ 通过 1,4-二甲氧基-2,5-二锂苯 与 $\mathrm{Cp}_{2} \mathrm{Zr}(\mathrm{Me}) \mathrm{Cl}$ 反应在苯环的对位引入两个 $\mathrm{Cp}_{2} \mathrm{ZrMe}$ 基 团, 在三甲基膦存在下经 $\beta-\mathrm{H}$ 消除反应合成了稳定的双 (锆-苯炔)配合物 26 和 27 , 其中 26 可以在反应体系中选 择性地结晶出来而得以与 27 分离(Eq. 2).
(1) Use of $\mathrm{Cp}_{2} \mathrm{Zr}(\mathrm{Me}) \mathrm{Cl}$

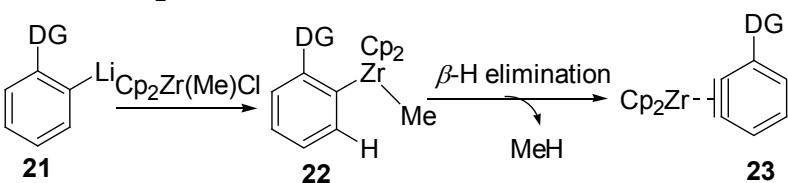

(2) Use of $\mathrm{Cp}_{2} \mathrm{Zr}(i-\mathrm{Bu}) \mathrm{Cl}$

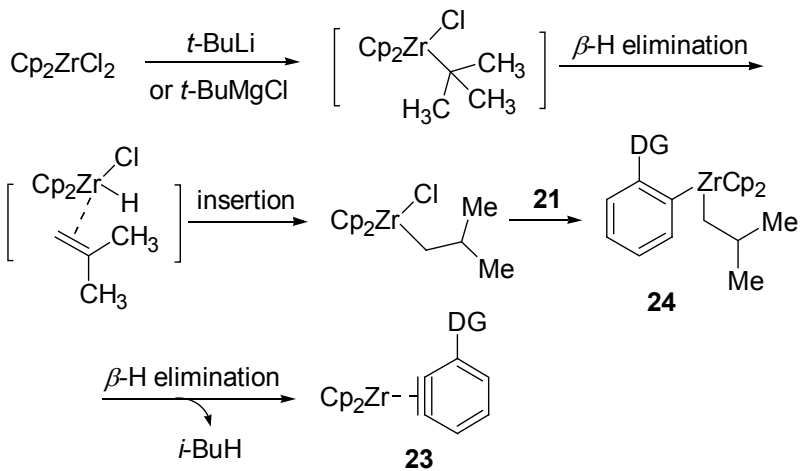

Scheme 6<smiles>CO[Z20](=O)c1cc(OC)c([Z20](C)=[Pt])cc1OC</smiles>

25<smiles></smiles>

26, major

\section{3 其它方法}

Mathey 小组 ${ }^{[15]}$ 通过形成甲基/芳基锆的方法合成了 膦杂苯的苯炔配合物 31. 即低价锆与溴代膦杂苯发生 配位/消除反应得到四价锆配合物 29, 29 经甲基化及 $\beta-\mathrm{H}$ 消除反应即可以较高收率得到锆-苯炔的二聚体 31 (Scheme 7).

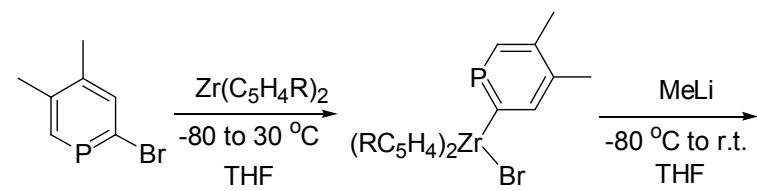

$28 \mathrm{R}=\mathrm{H}, \mathrm{Me}, \mathrm{SiMe}_{3}$

29
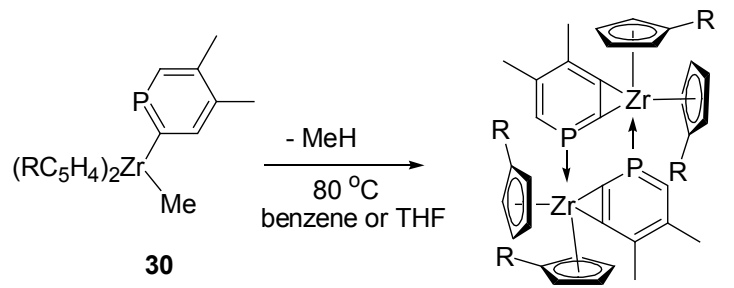

$31,65 \% \sim 70 \%$ 
Meunier 小组 ${ }^{[16]}$ 发现茂环上含叔丁基取代基的二芳 基二茂铅 32 在加热条件下原位生成锆-苯炔 33.33 中茂 环上叔丁基的 $\mathrm{C}-\mathrm{H}$ 键易被活化形成金属杂环 34.34 同 样可以作为一种新的锆-苯炔前体, 在特定的反应条件 下经芳环上的 $\beta$-H 消除反应得到两种锆-苯炔 33 和 35 (Scheme 8).

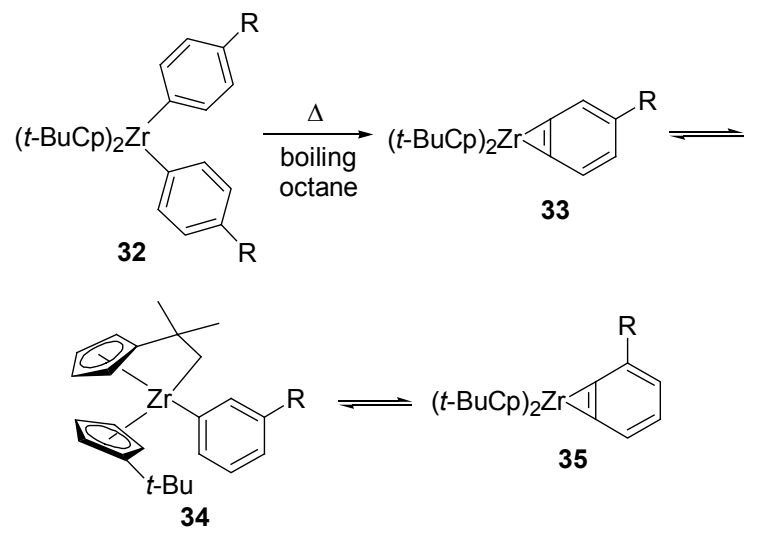

Scheme 8

\section{2 锆-苯炔参与的金属杂环的形成及后续反应}

\section{1 锆-苯炔与烯烃的反应及后续转化}

锆-苯炔与烯烃反应能够生成铅杂二氢狮如 $36^{[17]}$. Erker 小组 ${ }^{[17]}$ 发现锆杂二氢狮 36 与同时含有烯及苯炔配 位基团的锆配合物 37 存在平衡关系. 该现象可由下列 实验加以证实: 当向 36 中加入过量的降冰片烯进行热 解时, 该烯烃可以替换掉一分子己烯以定量的收率得到 锆杂环 39 (Scheme 9).

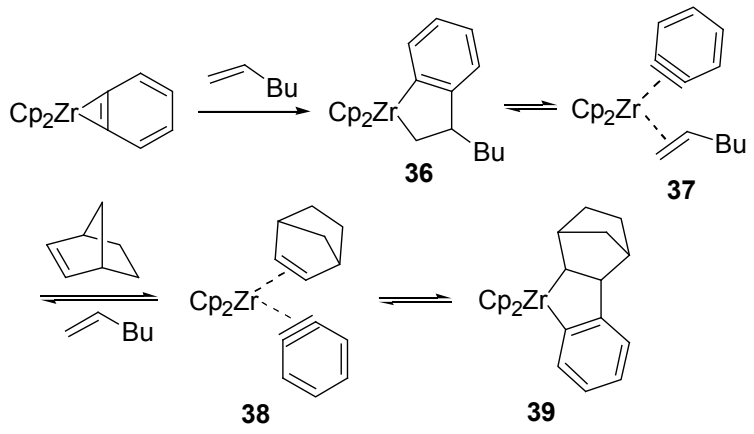

Scheme 9

Erker 小组 ${ }^{[18]}$ 还发现锆-苯炔与顺式或反式烯烃反 应可以分别得到单一的锆杂二氢狮 40 或 $\mathbf{4 1}$, 他们推测 40 为顺式锆杂环, 而 41 为反式铅杂环. 该反应表明偶 联反应过程为协同的立体专一性的历程(Scheme 10).

Meunier 小组 ${ }^{[19]}$ 研究了锆-苯炔与磷杂环戊烯的偶 联反应, 发现该反应具有良好的区域选择性. 由于磷与 锆的配位作用使得反应优先生成含磷基团处于 $\alpha$-位的 金属杂环 42.42 可与 $\mathrm{RPCl}_{2}$ 发生交换反应生成磷杂环 43, 43 可转化为更加稳定的双硫加和物 44 (Scheme 11).

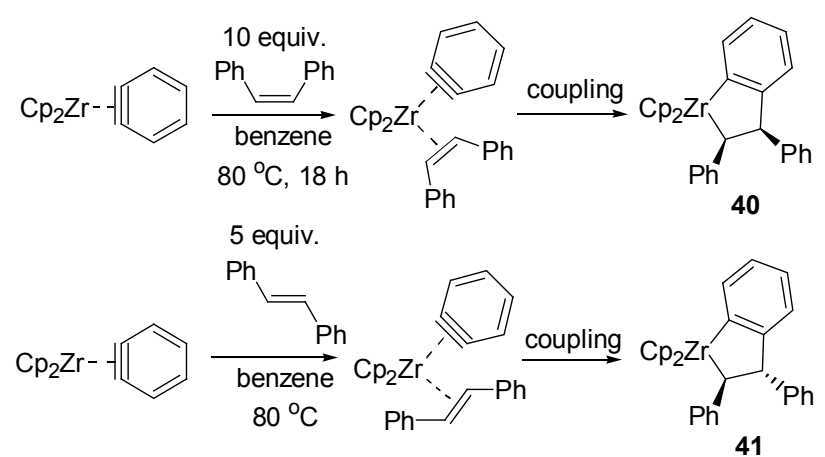

Scheme 10

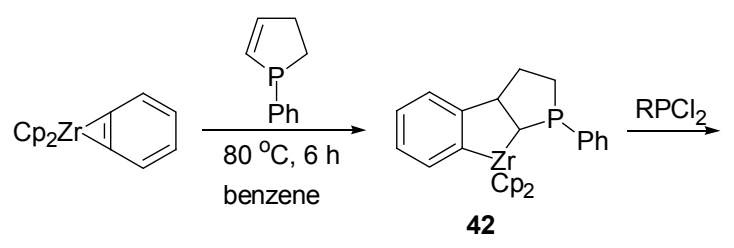

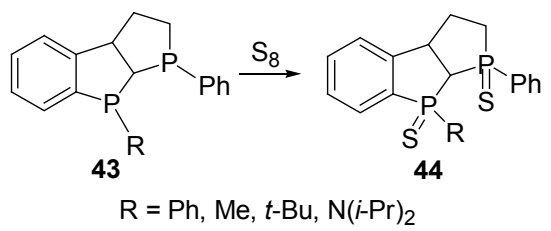

Scheme 11

Buchwald 小组 ${ }^{[0 \sim 12]}$ 利用锆-苯炔与烯烃的分子内偶 联反应合成了一系列多取代的吲哚啉或吲哚类化合物. 如化合物 45 经溴-锂交换后与 $\mathrm{Cp}_{2} \mathrm{ZrMeCl}$ 反应生成甲 基/芳基锆 46, 由 46 热解所形成的锆-苯炔中间体与分子 内烯烃偶联后生成锆杂环 $\mathbf{4 7 , 4 7}$ 经碘代以及脱除 HI 后 转化为 48 (Scheme 12). 48 在温和的条件下 $\left(85{ }^{\circ} \mathrm{C}\right.$, in toluene) 可与多种不饱和化合物发生 ene 反应得到官能 团化的吲哚 ${ }^{[9]}$ (Scheme 13).
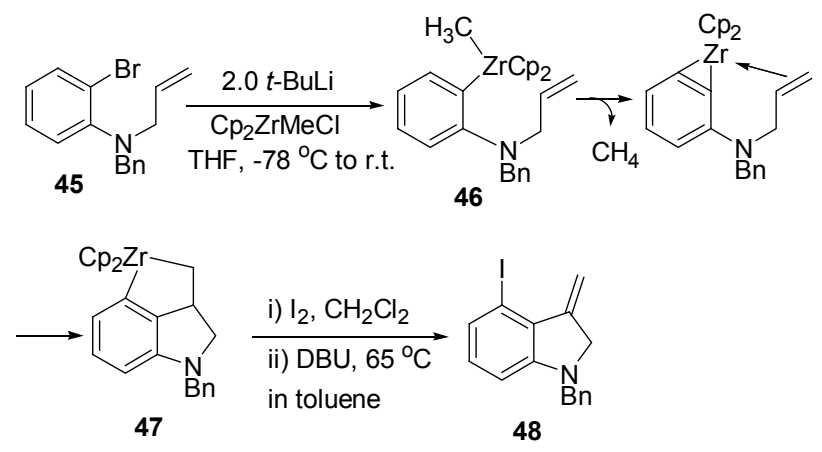

Scheme 12

该小组 ${ }^{[20]}$ 还将上述反应成功地应用于具有药用价 值的吲哚类分子的合成中. 例如, CC-1065 和 Duocarmy$\operatorname{cin} \mathrm{A}$ 等具有抗肿瘤活性的抗生素中具有相同的药效基 团, 即含有三元并环的螺环骨架 49 (Scheme 14), 但该 


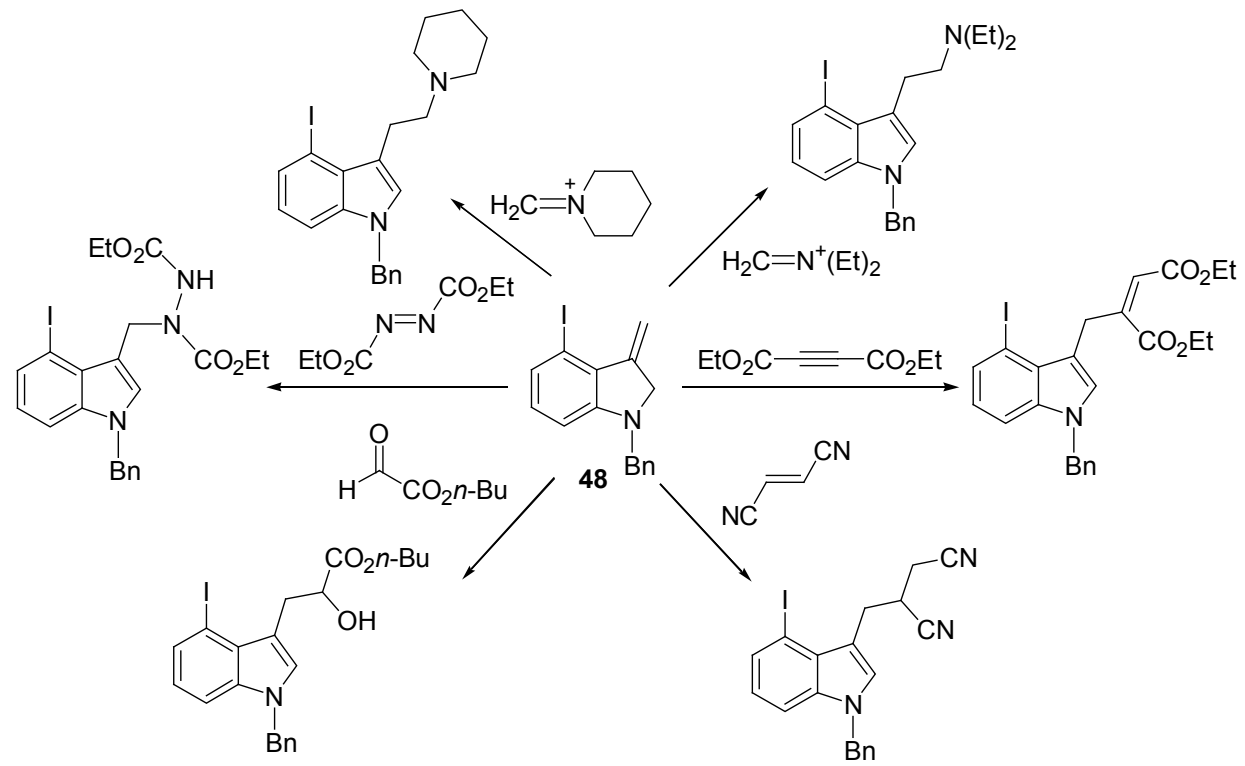

Scheme 13

结构的合成方法通常比较繁琐. Buchwald 小组 ${ }^{[21]}$ 从常见 的底物 50 出发, 经六步反应以形成锆-苯炔 52 和金属杂 环 53 为关键步骤方便地合成了该药效基团类似物 49 (Scheme 15).<smiles>CCOc1c2c(c3cc(C(=O)N4CC[C@@]5(C)C4=CC(=O)c4[nH]cc(C)c45)[nH]c3c1O)N(C(=O)c1cc3c4c(c(O)c(OC)c3[nH]1)N(C(N)=O)CC4)CC2</smiles>

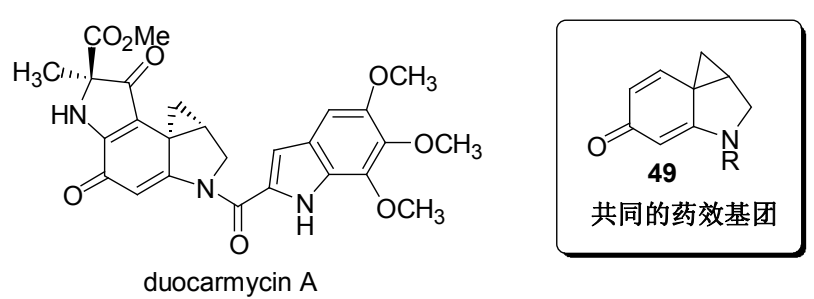

Scheme 14

他们还通过底物 55 经形成锆-苯炔及碘代反应得到 二碘化物 56, 56 经两步转化得到含有氨基片断的化合物 $\mathbf{5 8}^{[11]}, \mathbf{5 8}$ 经分子内 $\mathrm{C}-\mathrm{N}$ 键偶联以及脱除保护基就可以 合成天然产物去氢蟾酥碱 Dehydrobufotenine $(\mathbf{6 0})^{[12]}$ (Scheme 16).

2009 年, 席振峰小组 ${ }^{[22]}$ 报道了溴代烯丙基环丙烷 衍生物在低价锆作用下的碳一碳键断裂与重组反应. 例 如 61 在锆-苯炔作用下经过渡态 62 发生三元环的碳-碳 键断裂形成铅杂八元环 63,63 经水解得到产物 64 . 然而

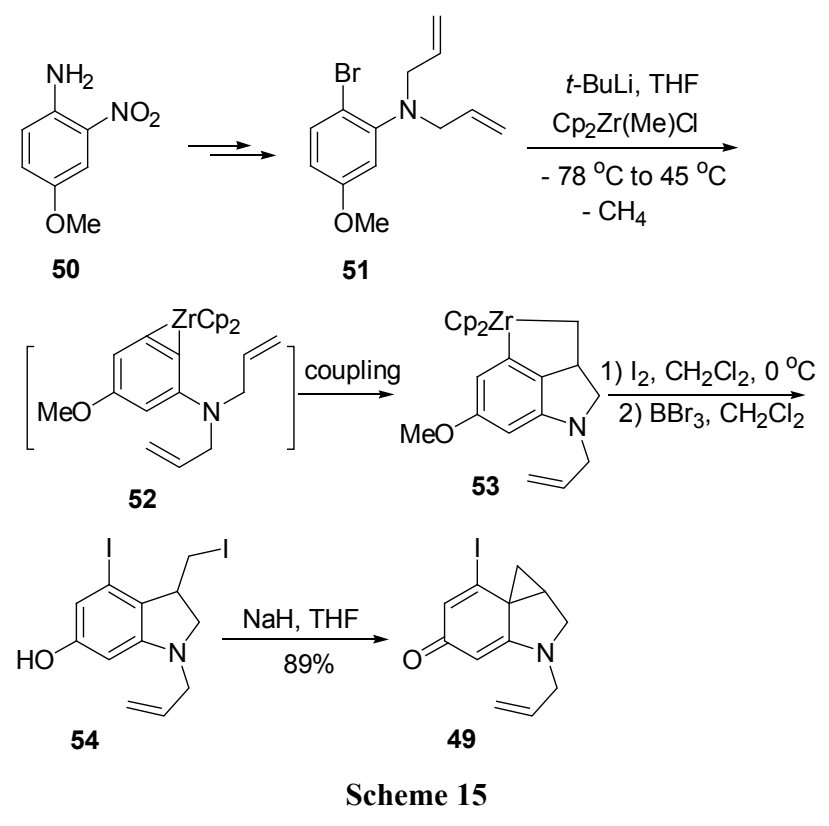

底物 61 在锆-丁烯作用下却经历完全不同的碳一碳键断 裂过程. 61 与低价锆配位后发生 $\gamma$-溴脱除反应生成三元 环中间体 65, 65 继续发生 $\beta$-碳断裂反应得到烷基锆物种 66, 66 与 $\mathrm{CO}$ 发生酰基化反应经水解得到醛 67 (Scheme 17).

Barluenga 小组 ${ }^{[23]}$ 报道了锆-苯炔与烯基溴的反应， 高立体选择性地合成了苯并环丁烯类化合物. 在该反应 中，首先芳基锂 68 与氯甲基二茂锆反应生成甲基/芳基 二茂锆 69, 69 经加热消除一分子甲烷后生成锆-苯炔 70 . 中间体 70 与顺式烯基溴反应时生成顺式锆杂环 71. 由 于 $\mathrm{R}^{1}$ 基团的位阻作用，烯基溴在插入 $\mathrm{Zr}-\mathrm{C}$ 键时选择性 地从远离 $\mathrm{R}^{1}$ 取代基的一端插入生成 $\alpha$-溴代锆杂二氢狮 


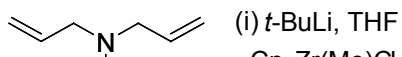

$$
\begin{aligned}
& { }_{\mathrm{OCH}_{3}}^{\mathrm{Br}}
\end{aligned}
$$

55
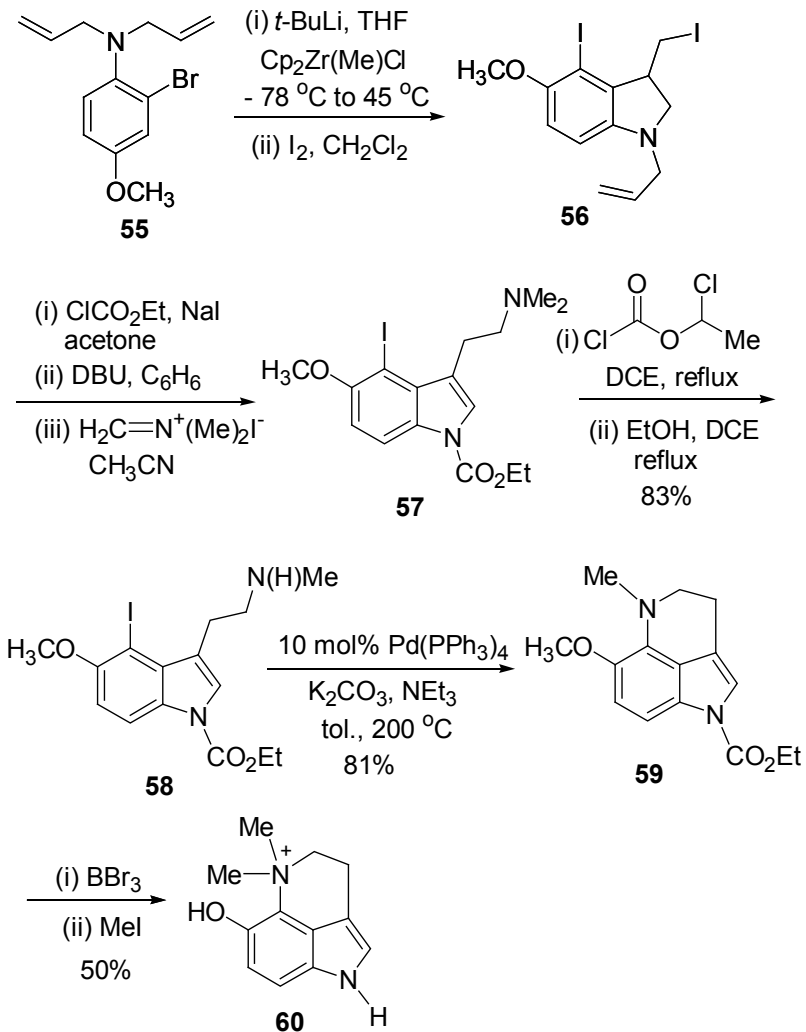

Dehydrobufotenine

\section{Scheme 16}

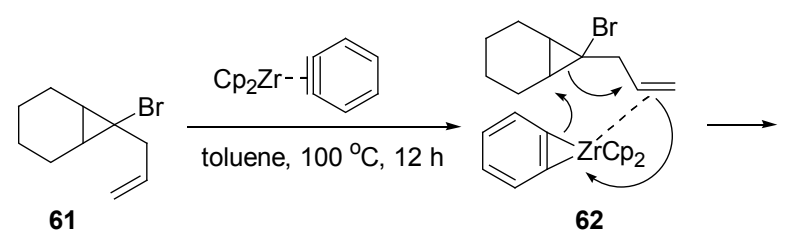

61

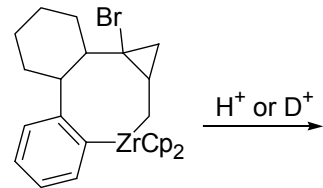

63

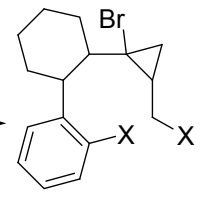

$64 \mathrm{X}=\mathrm{H}, 80 \%$

$$
X=D, 75 \%
$$

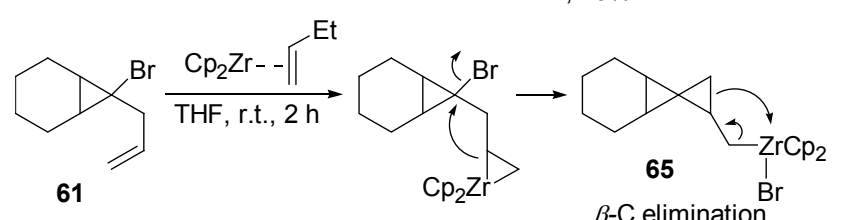

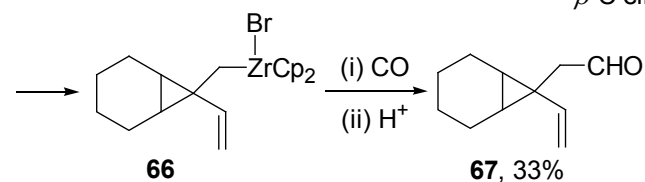

Scheme 17

71,71 经分子内类 $\mathrm{S}_{\mathrm{N}} 2$ 反应得到 $\alpha$-碳构型翻转的产物 72, 72 与亲电试剂反应可得到顺式苯并环丁烯 73 . 同样, 70 与反式烯基溴反应时可生成反式苯并环丁烯 76 (Scheme 18).

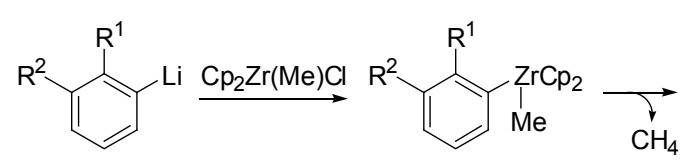

69

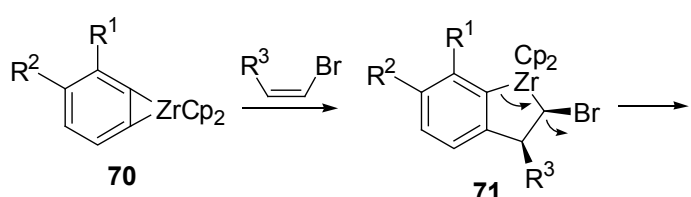

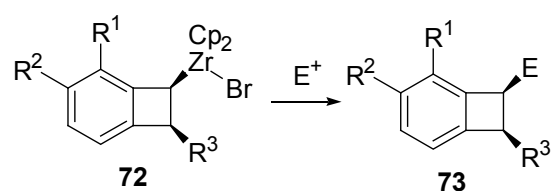

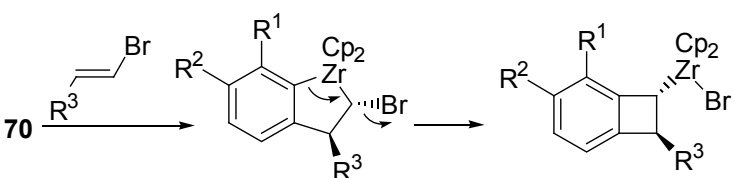<smiles>[R]c1ccc([R])c(C([Y])c2ccccc2)c1[R]</smiles>

75
$\mathrm{R}^{1}=\mathrm{Me}, \mathrm{Et}, \mathrm{MeO}, \mathrm{Ph} ; \mathrm{R}^{2}=\mathrm{H}$ or $\mathrm{R}^{1}=\mathrm{R}^{2}=(\mathrm{CH})_{4} ; \mathrm{R}^{3}=\mathrm{Me}$, decyl, Bn; $\mathrm{E}^{+}=\mathrm{H}_{2} \mathrm{O}, \mathrm{D}_{2} \mathrm{O}$, allyl chloride etc.

\section{Scheme 18}

\section{2 锆-苯炔与炔烃的反应及后续转化}

锆-苯炔与多种形式取代的炔烃进行偶联可以生成 种类丰富的锆杂环类化合物. 如 Buchwald 小组 ${ }^{[7]}$ 发展 的三甲基膦稳定的锆-苯炔 $\mathbf{2 0}$ 与简单炔烃如 3-已炔反应 可以得到锆杂荫 77 (Scheme 19). Regitz 小组 ${ }^{[2]]}$ 研究了 锆-苯炔与叔丁基取代的膦炔的反应，发现该反应可以 区域选择性地生成磷原子位于 $\beta$-位的锆杂磷杂环 78 (Scheme 19).

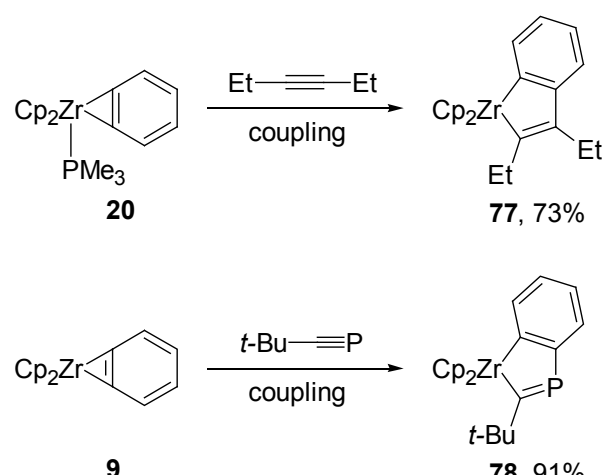

Scheme 19

双(锆-苯炔)络合物 26 可以与两分子的炔烃发生插 入反应，生成的产物以两个锆原子在苯环对位的锆杂环 80 为主 (Eq. 3) ${ }^{[8]}$. 


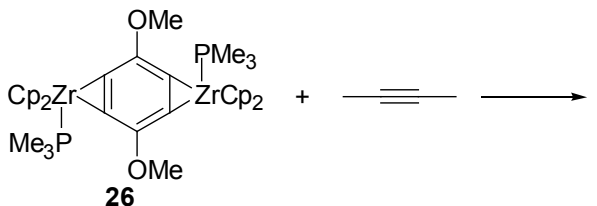

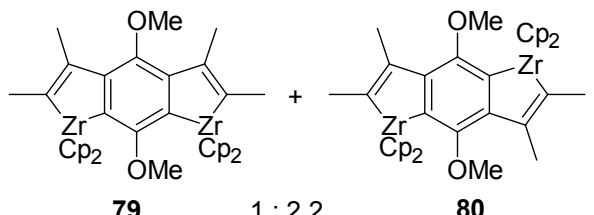

锆-苯炔与非对称取代的炔烃进行偶联时, 通常情 况下反应的区域选择性难以进行调控. Meunier 小组 ${ }^{[25]}$ 发现锆-苯炔与炔基膦反应时，可以选择性地生成含磷 基团处于 $\alpha$-位的锆杂狮 81,82 (Scheme 20). 该选择性与 前面所述生成磷杂环戊烯的区域选择性是一致的, 这也 再次说明了当底物中存在配位基团时, 往往会极大地影 响反应的选择性. 同样该类金属杂环如 81 可与 $\mathrm{PhSbCl}_{2}$ 或 $\mathrm{RPCl}_{2}$ 发生交换反应分别生成磷杂环 83 或锑杂环 85 . 其中 83 可转化为更加稳定的双硫加和物 84 .
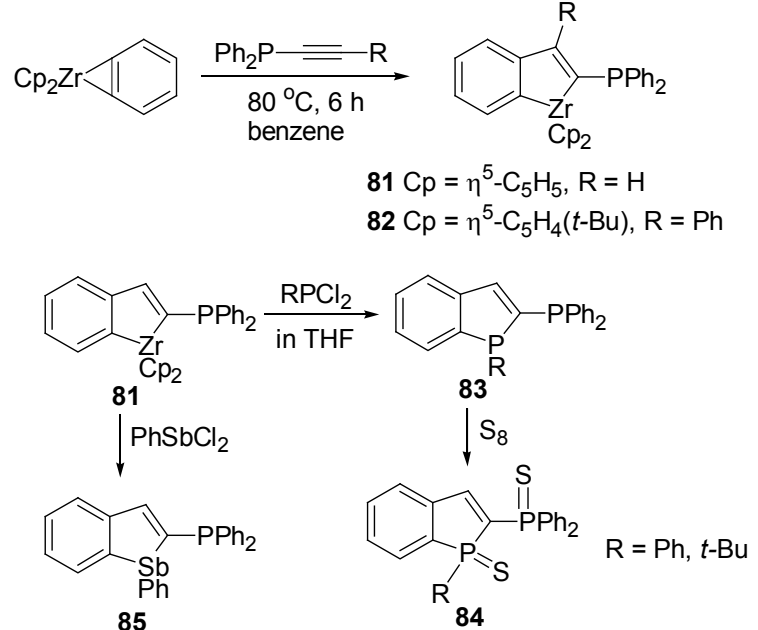

Scheme 20

Mathey 小组发展的锆-膦杂苯的苯炔配合物与经典 的锆-苯炔配合物具有类似的反应活性，也能与炔等不 饱和分子发生偶联反应. 如 Mathey 等 ${ }^{[15]}$ 发现 3,4-位含 有甲基取代基的膦杂苯的锆-苯炔前体 86 与炔基膦的反 应同样具有良好的区域选择性. 炔烃选择性地从位阻较 小的靠近磷原子的一端插入到 $\mathrm{Zr}-\mathrm{C}$ 键, 且由于磷与锆 的配位作用使得所生成的两种锆杂环 87 和 88 中以二苯 基膦基处于 $\alpha$-位的产物 87 为主(Scheme 21). 当炔烃为 三甲基硅基炔时，则优先生成三甲基硅基处于 $\alpha$-位的金 属杂环 90, 其水解产物 91 为空气敏感物质, 可以通过 转化为铇的配合物 92 来增加其稳定性.
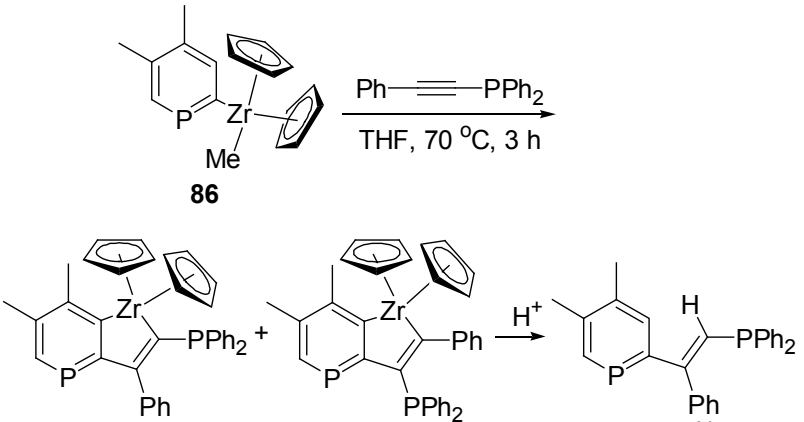

$87 \quad 95: 5 \quad 88$

$89,70 \%$
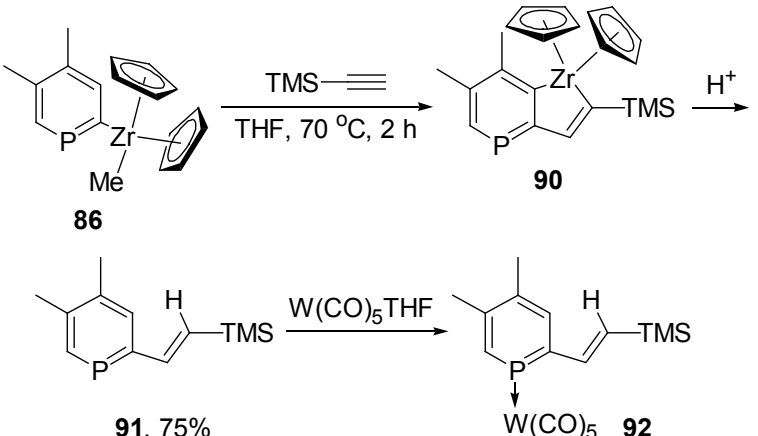

Scheme 21

含磷的锆杂狮 81 还能与炔、醛、二氧化碳、二硫 化碳、异腈酸酯等各种不饱和化合物发生 $[3+2]$ 环加成 反应，选择性地生成五配位的两性离子锆络合物 93 96 ${ }^{[26 ~ 29]}$ (Scheme 22). 此反应中磷取代基首先对底物进 行亲核进攻，然后在配位不饱和的锆金属中心发生环化 反应生成更加稳定的锆负离子物种. 另外, 含磷的锆杂 狮 81 还可以与叠氮化合物发生 $[3+1]$ 环加成反应，生成 叠氮和膦的螯合物 97 , 其受热脱去一分子氮气后生成 锆杂氮杂磷杂四元环 $98^{[30]}$.

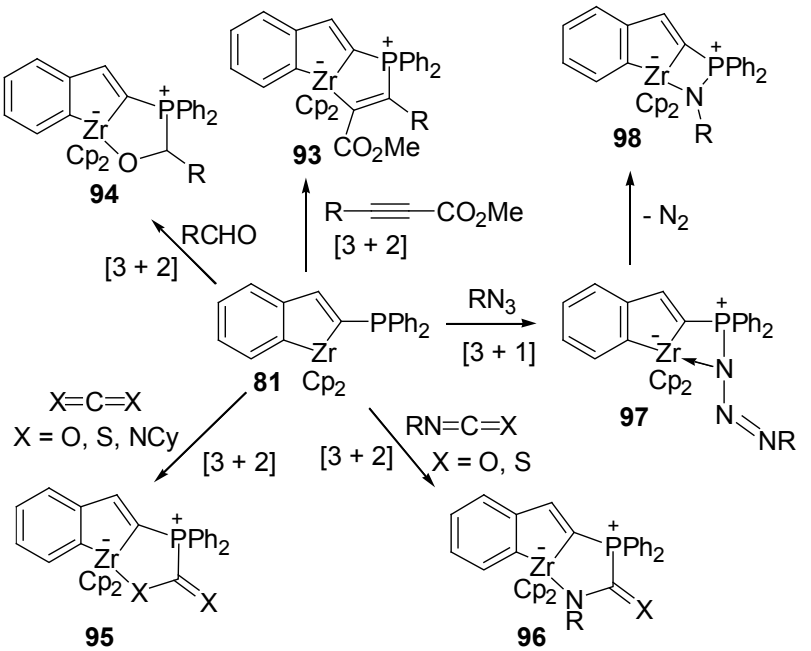

Scheme 22 
如果将炔基膦换成二炔基膦，再与锆-苯炔反应则 得到含磷杂四元环的中性锆配合物 100. 该反应中首先 锆-苯炔与二炔基膦反应生成锆杂环戊二烯中间体 99, 该中间体在高温下继续发生分子内环化反应生成四元 环产物 100 (Scheme 23) ${ }^{[31]} .100$ 与 $\mathrm{PhSbCl}_{2}$ 可发生金属转 移反应得到锑杂环 102.

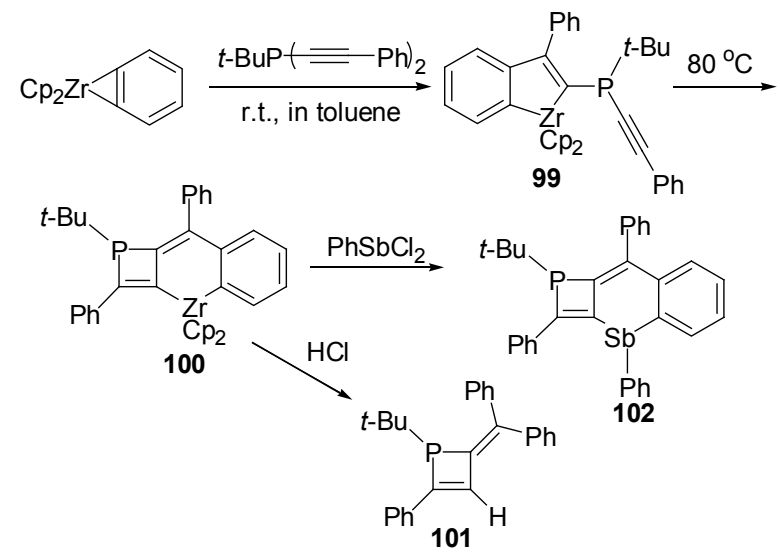

Scheme 23

Tilley 小组 ${ }^{[32]}$ 工仯地在锆-苯炔前体 106 的芳环对 位引入炔基片段, 利用分子间的交叉偶联反应实现了含 苯并锆杂环戊二烯单元的低聚物 105 的合成(Scheme 24). 该金属聚合物 105 被盐酸淬灭后生成有机聚合物 106. 105 与二氯化硫反应则可以得到主链中含有苯并噻 吩单元的聚合物 107.
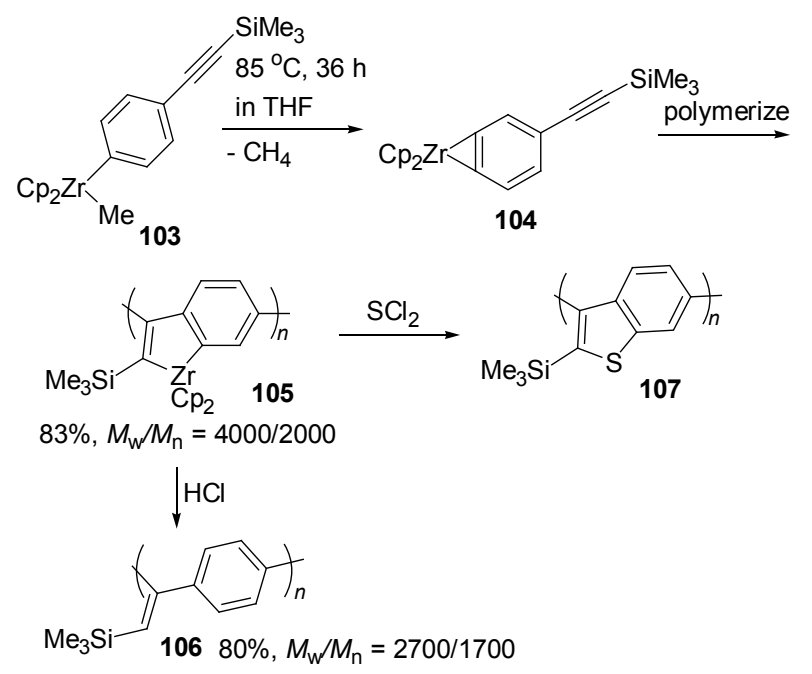

\section{Scheme 24}

Takahashi 小组 ${ }^{[33]}$ 发现, 锆-苯炔与普通炔烃偶联所 得到的锆杂狮 108 在 2 equiv. 溴化锌和催化量的四(三苯 基膦)钯存在下, 经过金属转移以及分子内环化等历程 最终得到多取代菜衍生物 109 110 (Scheme 25). 锆杂 狮的两个 $\mathrm{Zr}-\mathrm{C}\left(\mathrm{sp}^{2}\right)$ 键发生金属转移的顺序随取代基
$\mathrm{R}^{1}$ 而变化: 当 $\mathrm{R}^{1}$ 为芳基时，含有苯并环一端的 $\mathrm{Zr}-$ $\mathrm{C}\left(\mathrm{sp}^{2}\right)$ 键具有较高反应活性优先进行金属转移，选择性 地生成取代萮衍生物 109; 当 $\mathrm{R}^{1}$ 为烷基时，由于烯烃一 端的 $\mathrm{Zr}-\mathrm{C}\left(\mathrm{sp}^{2}\right)$ 键亲核性增加因此也能进行金属转移反 应，此时得到取代荎衍生物 109 和 110 的混合物. 席振 峰小组 ${ }^{[34]}$ 研究了在路易斯酸促进下铅杂狮与醛的反应, 合成了多取代狮类化合物 111 (Scheme 25). 该反应中醛 羰基上的碳一氧双键被切断, 醛羰基碳作为一个碳单元 与两个碳一碳参键环化形成了环戊二烯骨架. 席婵娟小 组 ${ }^{[35]}$ 发现铅杂狮 108 在氯化亚铜和芸醌的作用下, 可以 得到含有四元环的并环产物 112 (Scheme 25). 该反应可 能经金属转移以及氧化偶联过程产生苯并环丁二烯中 间体，随后发生分子间 $[4+2]$ 环加成反应而得到化合物 112.

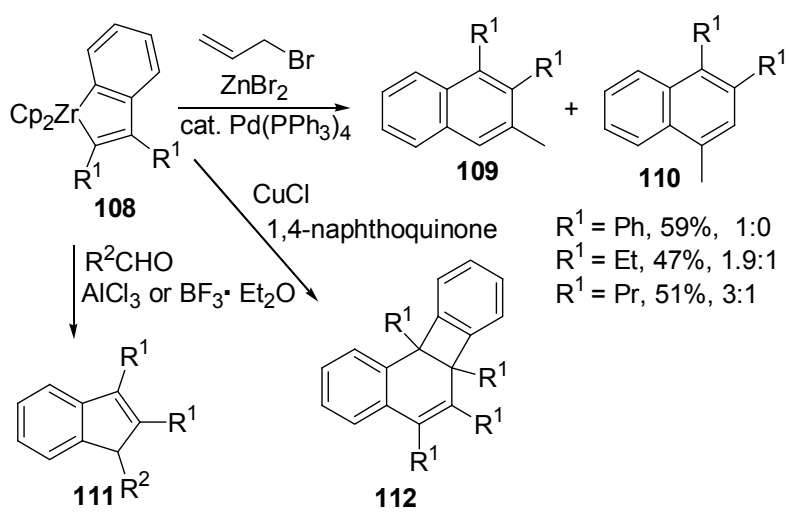

Scheme 25

2000 年, Meunier 小组 ${ }^{[36]}$ 发现锆-苯炔与苯基取代的 共轭双炔反应专一地生成了含有累积三烯结构的苯并 锆杂环庚四烯 114. 该反应首先生成 $\alpha$-炔基取代的锆杂 狮 113, 113 继续发生分子内扩环反应生成更加稳定的锆 杂环 114. X射线单晶衍射分析表明 114 中累积三烯呈扭 曲结构, 其构成锆杂环的各原子几乎共平面. 其中累积 三烯的中间双键对铅原子的配位作用使得该金属杂环 可以稳定存在. 2009 年该小组 ${ }^{[37]}$ 又发现三甲基硅基共轭 双炔与锆-苯炔反应时, 除了生成类似的含累积三烯片 段的金属杂环 115 以外还得到了 $\beta$-炔基取代的铅杂狮 116 (Scheme 26).

刘元红小组研究了多种形式取代的共轭双炔与锆苯炔的偶联反应. 发现该反应在盐酸的淬灭下生成顺式 烯炔类化合物 119. 对反应中间体的分离鉴定以及理论 计算研究表明, 在反应体系中主要生成含有累积三烯结 构的锆杂环 117, 其与 $\alpha$-炔基锆杂狮 118 存在平衡关系. 该类金属杂环与不同种类的酰腈化合物显示出截然不 同的反应性. 与芳基或烷基甲酰腈反应时, 羰基优先参 与反应，高立体选择性地得到用普通方法难以合成的顺 式累积三烯酮类产物 $\mathbf{1 2 0}$. 而与氨基或烷氧基甲酰腈反 


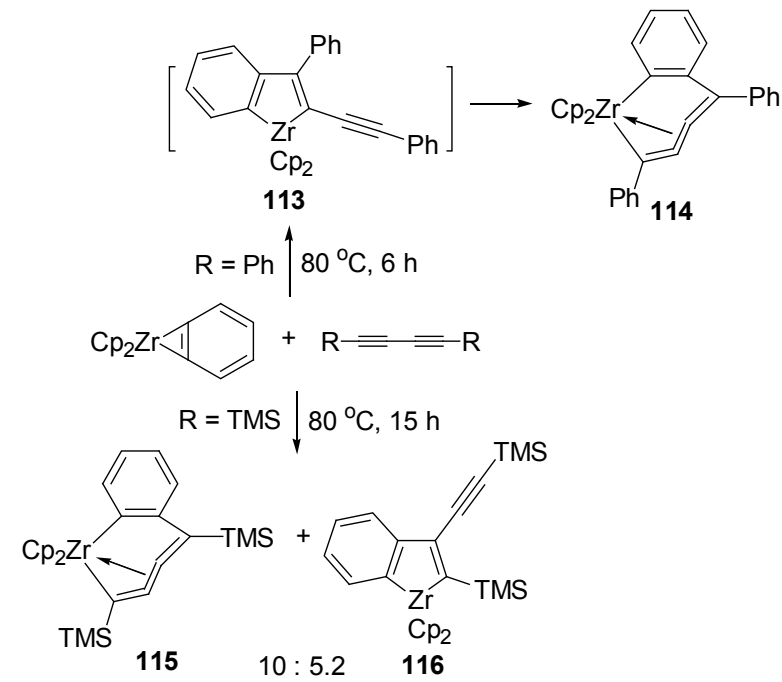

Scheme 26

应时，则是氰基首先参与反应，通过一系列复杂而奇特 的转化以良好的收率得到狮并 $[2,1-b]$ 吡咯类化合物 121, 其中涉及到芳环碳-氢键官能团化以及氢迁移(Scheme $27)^{[38]}$.

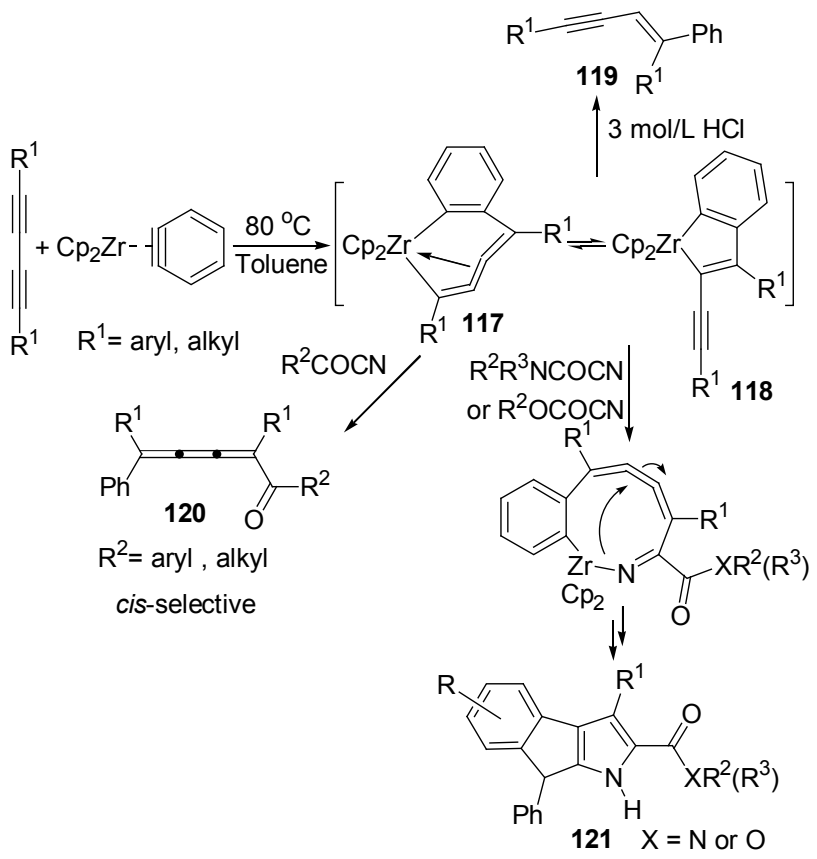

Scheme 27

该小组 ${ }^{[39]}$ 还发现锆杂环 117 可以在温和的条件下 与醛反应, 以中等至良好的收率选择性地得到顺式累积 三烯醇类化合物 122 (Scheme 28). 该反应中醛选择性地 与炔丙基锆一端发生加成反应, 而含苯基一端的锆-碳 键则未参与反应. 通过对可能的反应路径进行理论计算 表明, 该反应首先由稳定的七元铅杂累积三烯 117 异构 化为活性较高的五元炔基锆杂狮 118, 然后再与醛发生 $\mathrm{S}_{\mathrm{E}} 2^{\prime}$ 反应.

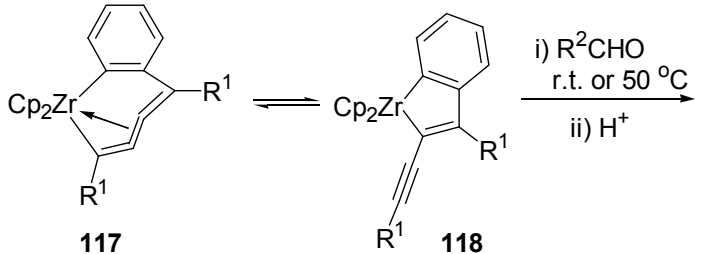

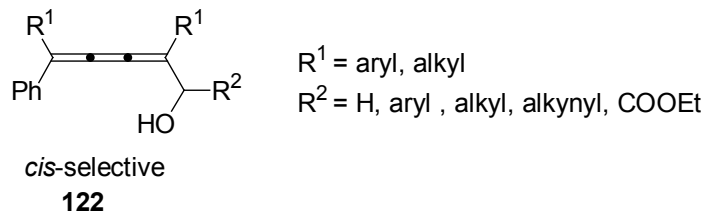

\section{Scheme 28}

\section{3 锆-苯炔与醛、酮、腈等的反应}

锆-苯炔与腈、酮等分子反应可以生成含杂原子的 锆杂环类化合物. 例如 Buchwald 小组 ${ }^{[7]}$ 发展的三甲基 膦稳定的锆-苯炔 20 与叔丁基腈或丙酮反应时, 腈基和 羰基均可以插入到锆-碳键中，分别生成相应的氮杂锆 杂狮 123 或氧杂锆杂环 124 (Scheme 29). 同样，无膦配 体稳定的锆-苯炔也可以与腈等分子发生类似的插入反 应 $^{[40]}$. 双(锆-苯炔)络合物 26 可以与两分子酮发生插入 反应，生成的产物以两个锆原子在间位的铅杂环 125 为 主(Scheme 29) ${ }^{[8]}$.<smiles>O=[Pt+]1N=C(C(Br)(Br)c2ccccc2)c2ccccc21</smiles><smiles>CC(C)(C)C(C)(C)N</smiles>
123, $94 \%$ $\mathrm{C} \equiv \mathrm{N}$ bond<smiles>[R16][Z10](=O)c1ccccc1</smiles>

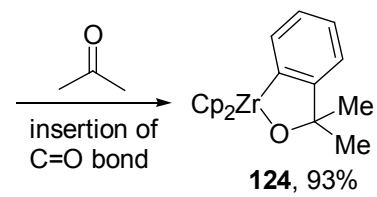
20
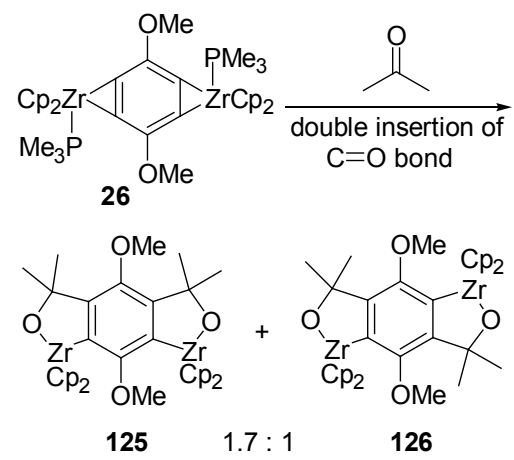

Scheme 29

通常 Friedel-Crafts 酰基化反应发生在芳环上含给 电子取代基的邻位或对位上. Buchwald 小组 ${ }^{[41]}$ 利用给电 子芳环的邻位诱导锂化反应形成相应的锆-苯炔中间体 实现了给电子芳环间位的直接酰化反应. 即给电子芳环 127 经邻位诱导锂化后与 $\mathrm{Cp}_{2} \mathrm{ZrRCl}$ 反应原位生成锆-芳 
炔配合物 130, 然后再与腈偶联生成五元锆杂环 131, 锆 杂环 131 经水解或碘解后生成间位酰基化的芳烃 132 或

133 (Scheme 30).

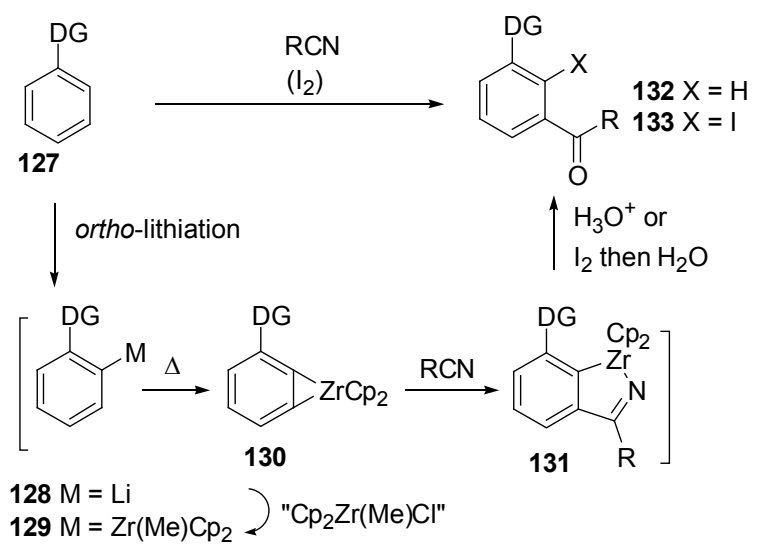

\section{Scheme 30}

\section{4 杂原子插入反应}

Meunier 小组 ${ }^{[16]}$ 发现硫族元素硒能够插入到锆-苯 炔的 $\mathrm{Zr}-\mathrm{C}$ 键中生成二硒杂锆杂环戊烯衍生物. 锆-苯 炔前体 34 生成锆-苯炔中间体时有两个不同的苯环邻位 $\mathrm{C}-\mathrm{H}$ 键可以发生消除反应, 所以得到两种锆-苯炔异构 体 33 和 35, 该中间体随即发生与硒的插入反应生成二 硒杂锆杂环戊烯 134 或 135 . 苯环上的取代基对反应的 选择性有较大影响. 例如，当 $\mathrm{R}$ 为甲氧基时，以 $60 \%$ $65 \%$ 的收率生成唯一的邻位取代的产物 $\mathbf{1 3 5}$; 当 $\mathrm{R}$ 为甲 基时, 得到的是 134 和 135 的混合物, 二者的比例为 60/40 (Scheme 31).
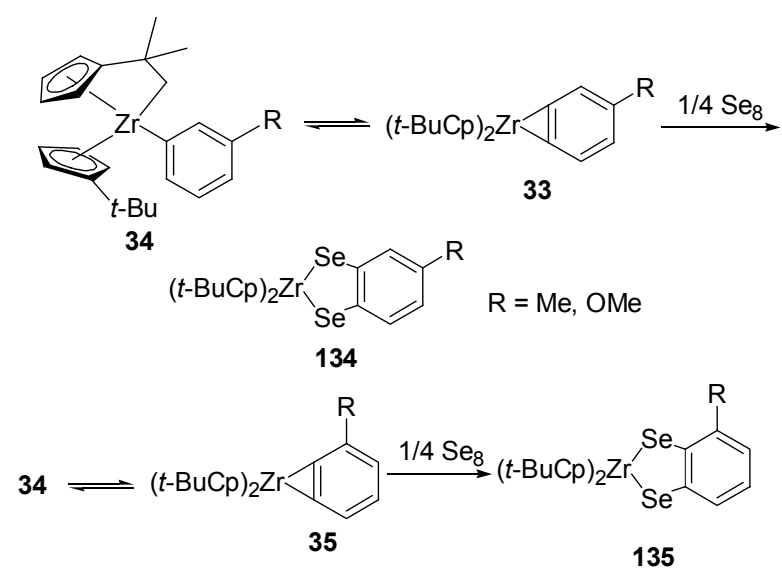

Scheme 31

锆-苯炔与锗硫双键 $\left[\mathrm{Me}_{2} \mathrm{Ge}=\mathrm{S}\right]^{[2]}$ 或氮磷双键 $\left[\mathrm{R}^{1} \mathrm{~N}=\mathrm{PNR}^{2} \mathrm{R}^{3}\right]^{[43]}$ 反应能够形成含相应杂原子的铅杂五 元环 136 或 137. 由于氧原子孤对电子与配位不饱和的 锆金属原子有较强的配位作用, 使得锆-苯炔与硼氧或 铝氧化合物可以发生金属转移反应生成含铝或嗍的双 金属配合物 138 $\sim$ 139 $^{[44]}$ (Scheme 32).

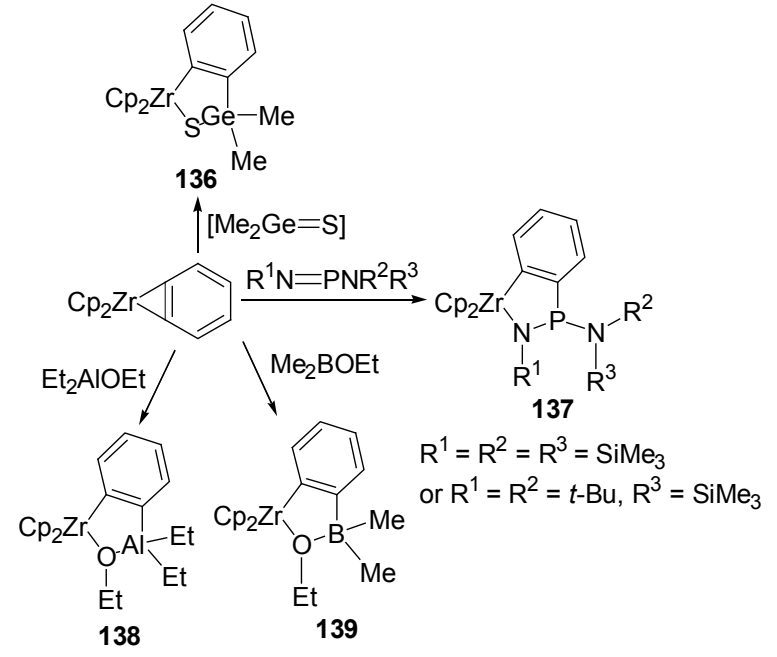

Scheme 32

双硒锆杂环 134 与四氯化锗进行金属转移反应时, 通过控制锆杂环 134 与四氯化锗的比例可以得到不同类 型的含有锗硒的杂环化合物. 例如 $\mathbf{1 3 4}$ 与 1 equiv.的四 氯化锗反应生成锗硒杂环 $\mathbf{1 4 0}$, 而与 0.5 equiv. 的四氯化 锗反应则得到螺环形式的锗硒杂环 141 (Scheme 33) ${ }^{[45]}$.

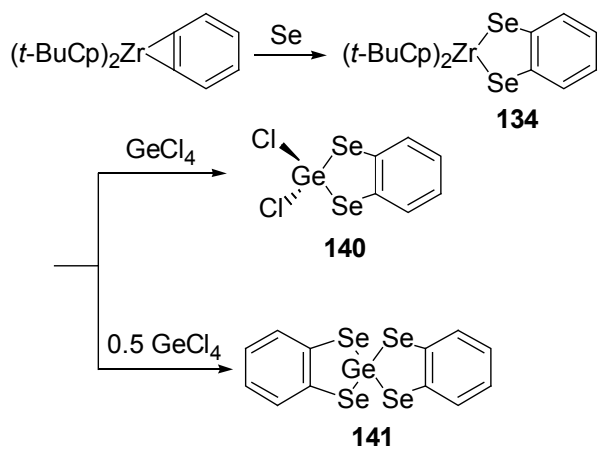

Scheme 33

双硒锆杂环 134 也可以用于合成硒杂醚类衍生物. 例如 134 先与 $t$ - $\mathrm{BuOK}$ 反应得到二硒钾盐 142,142 再与 二卤代物 $\mathrm{ClCH}_{2}\left(\mathrm{CH}_{2} \mathrm{OCH}_{2}\right)_{n} \mathrm{CH}_{2} \mathrm{Cl}$ 反应得到双氯化物 143,143 再与钾盐 142 反应便可得到含硒的大环冠醚类 化合物 144 (Scheme 34) ${ }^{[46]}$. 当冠醚 144 与重金属盐如 $\mathrm{HgI}_{2}$ 进行络合反应时, 得到的是较软的硒与录配位的络 合物 145 , 而氧原子并未参与配位. 该类金属冠醚有望 用于重金属离子的检测或提取.

\section{3 结论与展望}

锆-苯炔可由其前体二芳基二茂锆或烷基/芳基二茂 锆等在加热条件下经 $\beta$ - $\mathrm{H}$ 瞳取反应得到. 锆-苯炔与烯 烃、炔烃、醛、酮等不饱和底物反应时，通过调节底物 的取代基、化学计量比、配体结构以及反应条件等因素, 

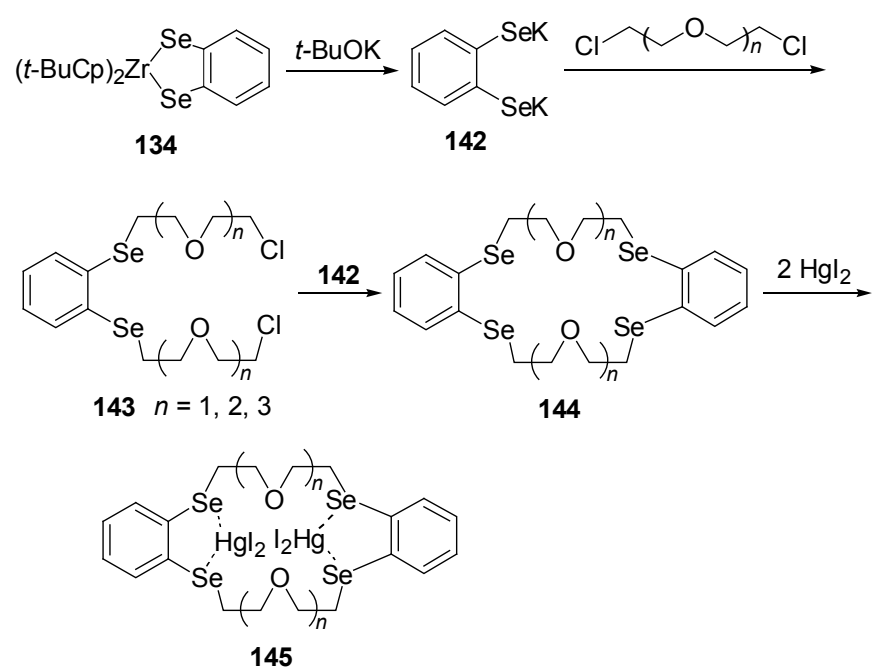

Scheme 34

可以得到多种形式的锆杂环类金属有机化合物. 锆杂环 可以与多种亲电试剂反应得到结构丰富的有机化合物. 锆-苯炔参与的金属杂环的形成及其后续反应已被成功 地应用到吲哚等天然产物合成中. 但是目前锆-苯炔的 制备方法仍然有限, 锆-苯炔化学多数集中于其与简单 的不饱和底物进行偶联所形成的金属杂环的分离鉴定 与结构表征, 而后续反应研究较少, 尤其是锆-苯炔与 共轭双炔、多炔等配位位点较多的不饱和底物的反应及 其所形成的金属杂环在有机合成的应用还有待进一步 研究.

\section{References}

[1] (a) Kitamura, T. Aust. J. Chem. 2010, 63, 987.

(b) Wentrup, C. Aust. J. Chem. 2010, 63, 979.

[2] (a) Wu, Q.; Li, B.; Shi, C.; Chen, Y. Chin. J. Synth. Chem. 2007, 15, 111 (in Chinese).

(仵清春, 李保山, 石常青, 陈彦逍, 合成化学, 2007, 15, 111.)

(b) Xing, Q.; Xu, R.; Zhou, Z.; Pei, W. Basic Organic Chemistry, 2nd ed., Higher Education Press, Beijing, 1994 (in Chinese).

(邢其毅, 徐瑞秋, 周政, 裴伟伟, 基础有机化学 (第二版), 高等 教育出版社, 北京, 1994.)

(c) Matsumoto, T.; Hosoya, T.; Katsuki, M.; Suzuki, K. Tetrahedron Lett. 1991, 32, 6735.

(d) Schlosser, M.; Castagnetti, E. Eur. J. Org. Chem. 2001, 3991.

(e) Caster, K. C.; Keck, C. G.; Walls, R. D. J. Org. Chem. 2001, 66, 2932.

(f) Vagin, S. I.; Frickenschmidt, A.; Kammerer, B.; Hanack, M. Chem. Eur. J. 2007, 13, 985.

(g) Kitamura, T.; Yamane, M. J. Chem. Soc., Chem. Commun. 1995, 983.

(h) Himeshima, Y.; Sonoda, T.; Kobayashi, H. Chem. Lett. 1983, 1211.

[3] (a) Majoral, J.-P.; Igau, A.; Cadierno, V.; Zablocka, M. Top. Curr. Chem. 2002, 220, 53.

(b) Majoral, J.-P.; Meunier, P.; Igau, A.; Pirio, N.; Zablocka, M.; Skowronska, A.; Bredeau, S. Coord. Chem. Rev. 1998, 178 180, 145. (c) Buchwald, S. L.; Nielsen, R. B. Chem. Rev. 1988, 88, 1047.

(d) Jones, W. M.; Klosin, J. ChemInform 1998, 29, 1522.

[4] Samuel, E.; Rausch, M. D. J. Am. Chem. Soc. 1973, 95, 6263.

[5] Clegg, W.; Horsburgh, L.; Lindsaym D. M.; Mulvey, R. E. Acta Crystallogr. 1998, C54, 315.

[6] Erker, G. J. Organomet. Chem. 1977, 134, 189.

[7] Buchwald, S. L.; Watson, B. T. J. Am. Chem. Soc. 1986, 108, 7411.

[8] Buchwald, S. L.; Lucas, E. A.; Dewan, J. C. J. Am. Chem. Soc. 1987, 109, 4396.

[9] Tidwell, J. H.; Senn, D. R.; Buchwald, S. L. J. Am. Chem. Soc. 1991, 113, 4685.

[10] Tidwell, J. H.; Peat, A. J.; Buchwald, S. L. J. Org. Chem. 1994, 59, 7164.

[11] Tidwell, J. H.; Buchwald, S. L. J. Am. Chem. Soc. 1994, 116, 11797.

[12] Peat, A. J.; Buchwald, S. L. J. Am. Chem. Soc. 1996, 118, 1028.

[13] Barr, K. J.; Waston, B. T.; Buchwald, S. L. Tetrahedron Lett. 1991, $32,5465$.

[14] (a) Negishi, E.; Miller, J. A.; Yoshida, T. Tetrahedron Lett. 1984, $25,3407$.

(b) Swanson, D. R.; Nguyen, T.; Noda, Y.; Negishi, E. J. Org. Chem. 1991, 56, 2590.

[15] Rosa, P.; Floch, P. L.; Ricard, L.; Mathey, F. J. Am. Chem. Soc. 1997, 119, 9417.

[16] Legrand, C.; Meunier, P.; Petersen, J. L.; Tavares, P.; Bodiguel, J.; Gautheron, B.; Dousse, G. Organometallics 1995, 14, 162.

[17] Erker, G.; Kropp, K. J. Am. Chem. Soc. 1979, 101, 3659.

[18] Kropp, K.; Erker, G. Organometallics 1982, 1, 1246.

[19] Zablocka, M.; Cénac, N.; Igau, A.; Donnadieu, B.; Majoral, J. P.; Skowronska, A.; Meunier, P. Organometallics 1996, 15, 5436.

[20] (a) Hanka, L. J.; Dietz, A.; Gerpheide, S. A.; Kuentzel, S. L.; Martin, D. G. J. Antibiot. 1978, 31, 1211.

(b) Chidester, C. G.; Krueger, W. C.; Mizsak, S. A.; Duchamp, D. J.; Martin, D. G. J. Am. Chem. Soc. 1981, 103, 7629.

[21] Tidwell, J. H.; Buchwald, S. L. J. Org. Chem. 1992, 57, 6380.

[22] Wang, C.; Deng, L.; Yan, J.; Wang, H.; Luo, Q.; Zhang, W.; Xi, Z. Chem. Commun. 2009, 4414.

[23] Barluenga, J.; Calleja, J.; Antón, M. J.; Álvarez-Rodrigo, L.; Rodríguez, F.; Fananás, F. J. Org. Lett. 2008, 10, 4469.

[24] Ma, X.-B.; Regitz, M. Synthesis 1995, 667.

[25] Miquel, Y.; Igau, A.; Donnadieu, B.; Majoral, J.-P.; Dupuis, L.; Pirio, N.; Meunier, P. Chem. Commun. 1997, 279.

[26] Miquel, Y.; Cadierno, V.; Donnadieu, B.; Igau, A.; Majoral, J.-P. Organometallics 2000, 19, 54

[27] Cadierno, V.; Igau, A.; Donnadieu, B.; Caminade, A. M.; Majoral, J.-P. Organometallics 1999, 18, 1580.

[28] Cadierno, V.; Zablocka, M.; Donnadieu, B.; Igau, A.; Majoral, J.-P. Organometallics 1999, 18, 1882.

[29] Miquel, Y.; Igau, A.; Donnadieu, B.; Majoral, J.-P.; Pirio, N.; Meunier, P. J. Am. Chem. Soc. 1998, 120, 3504

[30] Cadierno, V.; Zablocka, M.; Donnadieu, B.; Igau, A.; Majoral, J.-P.; Skowronska, A. Chem. Eur. J. 2000, 6, 345.

[31] Dupuis, L.; Pirio, N.; Meunie, P.; Igau, A.; Donnadieu, B.; Majoral, J.-P. Angew. Chem., Int. Ed. 1997, 36, 987.

[32] Mao, S. S. H.; Tilley, T. D. J. Organomet. Chem. 1996, 521, 425.

[33] Duan, Z.; Nakajima, K.; Takahashi, T. Chem. Commun. 2001, 1672.

[34] Zhao, C.; Li, P.; Cao, X.; Xi, Z. Chem. Eur. J. 2002, 8, 4292.

[35] Chen, C.; Xi, C.; Liu, Y.; Hong, X. J. Org. Chem. 2006, 71, 5373.

[36] Bredeau, S.; Delmas, G.; Pirio, N.; Richard, P.; Donnadieu, B.; Meunier, P. Organometallics 2000, 19, 4463.

[37] Bredeau, S.; Ortega, E.; Delmas, G.; Richard, P.; Frohlich, R.; 
Donnadieu, B.; Kehr, G.; Pirio, N.; Erker, G.; Meunier, P. Organometallics 2009, 28, 181.

[38] Fu, X.; Chen, J.; Li, G.; Liu, Y. Angew. Chem., Int. Ed. 2009, 48, 5500.

[39] Fu, X.; Liu, Y.; Li, Y. Organometallics 2010, 29, 3012.

[40] Buchwald, S. L.; Sayers, A.; Watson, B. T.; Dewan, J. C. Tetrahedron Lett. 1987, 28, 3245.

[41] Akai, S.; Peat, A. J.; Buchwald, S. L. J. Am. Chem. Soc. 1998, 120, 9119.

[42] Bodlguel, J.; Meunier, P.; Kubicki, M. M.; Richard, P.; Gautheron,
B.; Dousse, G.; Lavayssière, H.; Satgé, J. Organometallics 1992, $11,1423$.

[43] Dupuis, L.; Pirio, N.; Meunier, P.; Gautheron, B.; Mahieu, A.; Igau, A.; Majoral, J. P. Bull. Soc. Chim. Fr. 1996, 133, 611.

[44] Rege, F. M. G.; Davis, W. M.; Buchwald, S. L. Organometallics 1995, 14, 4799.

[45] Tavarès, P.; Meunier, P.; Kubicki, M. M.; Gautheron, B.; Dousse, G.; Lavayssière, H.; Satgé, J. Heteroat. Chem. 1993, 4, 383.

[46] Mazouz, A.; Meunier, P.; Kubicki, M. M.; Hanquet, B.; Amardeil, R.; Bornet, C.; Zahidi, A. J. Chem. Soc., Dalton Trans. 1997, 1043.

(Li, L.) 